\title{
Endophytic bacteria of the Bacillus induce resistance of potato plants to viruses
}

\author{
Burkhanova G.*, Sorokan A., Cherepanova E., Maksimov I. \\ Institute of Biochemistry and Genetics - Subdivision of the Ufa Federal Research Centre of the Russian \\ Academy of Sciences, Ufa, Russia \\ *email: guzel_mur@mail.ru
}

Inheritance of potato resistance to viruses can be determined by the activity of protective proteins and enzymes involved in nonspecific mechanisms of plant resistance, such as ribonuclease (RNase). In our work, it was shown that in potato plants treated with bacterial cell suspensions of the $B$. subtilis 26D and B. thuringiensis B-6066 strains, the RNase activity significantly increased and the viral RNA content decreased upon infection with PVX viruses. The same effect was observed when plants were treated with the $B$. subtilis TS2 separately and together with $B$. thuringiensis B-5351 against PVS; and bacterial strains B. subtilis TS2, B. subtilis 26D and B. subtilis Tt12 against PVY; $B$. thuringiensis $\mathrm{B}-5351$ against $\mathrm{M}$. Thus, the activation of RNases induced by inoculation with endophytes is virus-specific. Treatment of potato plants with B. thuringiensis B-6066 bacteria increased the expression of the PR1, PR4 genes; B. subtilis TS2 - PR1, PR4; B. thuringiensis B-5351 and B. subtilis STl-7 - PR1, PR4, PR6, PR10; B. subtilis TS2 and B. subtilis 26D - PR1, PR4, PR6, PR9, PR10, when infected with viruses. Co-inoculation of plants with a preparation of a combination of bacteria $B$. thuringiensis B-5351 and B. subtilis TS2 and B. thuringiensis B-5351 and $B$. subtilis $26 \mathrm{D}$ led to a significant increase in the level of expression of all studied genes upon infection with the PVS or PVM virus compared to the control. The combination of different endophytic bacterial strains can promote activation of resistance reactions in potato plants. Thus, these bacterial strains, which have high RNase activity, activate plant RNases and expression PR-proteins, leads to the development of plant induced systemic resistance and can be proposed for creating new microbiological compositions for combating viral plant infections.

Acknowledgments: The work is supported by Department of science and technology (DST) of the Government of India No. 19-46-02004. 